Notfall Rettungsmed 2012 $\cdot 15: 287-288$

DOI 10.1007/s10049-011-1503-3

Online publiziert: 16. Juni 2012

(c) Springer-Verlag 2012

U. Kreimeier ${ }^{1} \cdot$ P. Sefrin ${ }^{2}$

${ }^{1}$ Klinik für Anaesthesiologie, Klinikum der Universität München

${ }^{2}$ Arbeitsgemeinschaft der in Bayern tätigen Notärzte (agbn), Würzburg

\title{
Schnittstellen in der Notfallmedizin
}

tegrierter Leitstellen wurde die zentrale Koordination eines Notfalleinsatzes in die Hände einer „Logistikzentrale" gelegt. Der Leitstelle obliegt es, das geeignete Rettungsmittel zu disponieren; vielerorts übernimmt sie die Zuweisung einer geeigneten Klinik. Derartige Strukturen haben ihre Leistungsfähigkeit anhand von Kenngrößen zu belegen, wie z. B. die Zahl der entgegengenommenen Anrufe, Anrufwartezeit oder Dispositionszeit.

Die Autoren von Kaufmann und Kanz analysieren die vielfältigen Aufgaben einer Leitstelle. Neben den in einigen Bundesländern durch die reine Fahrzeit des Rettungsdienstes vom Gesetzgeber vorgegebene Hilfsfrist gewinnt die Qualität der Disposition im Hinblick auf die verschiedenen Schnittstellen zunehmend an Bedeutung: Standardisierte Notrufabfrageprotokolle, Rückmeldesysteme und ein Behandlungskapazitätsnachweis stellen Herausforderungen an eine moderne Leitstelle dar, die dazu dienen, den Patienten der präklinischen Verdachtsdiagnose entsprechend schnellstmöglich einer adäquaten Therapie zuzufuhren.

\section{》) Zeit- und Informationsverlust können zu einem Gefährdungs- potenzial in der Versorgungs- kette führen}

heit: umfangreiche und zielführende

Erstdiagnostik zur Sicherung der notwendigen Kausaltherapie,.

- aus der Sicht der (intensivmedizinischen) Weiterversorgung: konkrete medizinische und pflegerische Behandlungsvorgaben.

Das Leitthema dieser Ausgabe greift drei Elemente der Versorgung von Notfallpatienten heraus. Durch die Einführung In-
Zeit- und Informationsverlust können neben inkompatiblen Gerätschaften, verschiedenen Behandlungsempfehlungen und Hierarchien zu einem nicht unerheblichen Gefährdungspotenzial in der Versorgungskette gerade schwerstverletzter Patienten führen. Lendemans betont in seigung neben der reinen Prozessoptimienem Beitrag, dass bei der Notfallversor- rung die Minimierung von Fehlern in der Medizin ein zentrales Thema darstellt. Die Bildung von Traumanetzwerken dient einer Standardisierung bei der Versorgung und schafft zudem durch Kooperation verschiedener Kliniken auch erweiterte Behandlungsoptionen und -kapazitäten. Der Autor vertritt die Ansicht, dass Schnittstellen im Traumanetzwerk zwar potenzielle Schwachpunkte sind, durch konkrete Kurskonzepte aber durchaus trainierbar sind.

Zur Vermeidung von Problemen können heute Netzwerke für die wesentlichen Notfalldiagnosen nützlich sein. Mit ihnen gelingt es nicht nur, eine Vernetzung der differenten Bereiche, sondern auch einen Beitrag zu einem optimierten Austausch von Ressourcen zu erreichen, dessen Ziel die Schaffung von Grundlagen für eine verbesserte Patientenversorgung sein sollte. Zur Vermeidung von Problemen sind klare Zuständigkeiten, gesicherte und offene Kommunikation und gegenseitige Akzeptanz erforderlich. In ihrem Beitrag über Myokardinfarkt-Netzwerke stellen die Kollegen von Scheidt und Maier die zentrale Bedeutung der leitliniengerechten Festlegung von präklinischen und klinischen Standards für die Versorgung von Herzinfarktpatienten heraus. Die Erfassung der relevanten Versorgungszeiten bedeutet für alle Beteiligten Transparenz hinsichtlich der Teilschritte von der Alarmierung bis zur endgültigen Therapie. Grundvoraussetzung sind die gemeinsame Festlegung klarer Therapiepfade und Standards sowie deren Anpassung an die regionalen Gegebenheiten.

Bei Traumapatienten funktioniert die Verzahnung zwischen Präklinik und Klinik dank konsentierter Versorgungskonzepte (ATLS, ITLS, PHTLS). Bei internistischen und neurologischen Notfällen scheint es noch häufiger zu Kommuni- 
kations- und Schnittstellenproblemen zu kommen. Aber auch hier haben sich inzwischen exemplarisch Netzwerke für die Versorgung von Herzinfarkt- und Schlaganfallpatienten etabliert. Die Problematik liegt auch in einer vergleichsweise schwierigen Diagnostik am Notfallort. Eine sich möglicherweise konkret abzeichnende Option zur Verringerung des Informationsverlusts mit Übergabe des Patienten stellt die drahtlose Übertragung von Befunden vom Rettungsdienst an die aufnehmende Klinik dar.

Netzwerkstrukturen erscheinen sinnvoll und attraktiv im Hinblick auf eine regionale Absprache zum Vorgehen bei notfallmedizinisch und gesundheitspolitisch sowie ökonomisch wichtigen Krankheitsbildern. Eine transparente Rückkopplung an alle Beteiligten deckt Verbesserungspotenziale auf und dient zur Optimierung der Behandlungsstrategie. Prozessabläufe, Schnittstellenkommunikation und Teamarbeit sollten von allen an den Einzelprozessen Beteiligten regelmäßig trainiert werden, um gerade im Hinblick auf die notfallmedizinische Versorgung eine optimierte Behandlungsstrategie zu etablieren.

Ihre

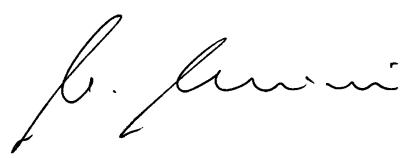

Uwe Kreimeier

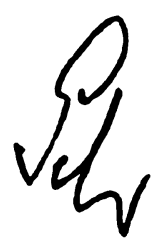

Peter Sefrin

\section{Korrespondenzadresse}

\section{Prof. Dr. U. Kreimeier}

Klinik für Anaesthesiologie,

Klinikum der Universität München

Nussbaumstraße 20, 80336 München

Uwe.Kreimeier@med.uni-muenchen.de

\section{Prof. Dr. P. Sefrin}

Arbeitsgemeinschaft

der in Bayern tätigen Notärzte (agbn)

Sandweg 11, 97078 Würzburg

sefrin@agbn.de

\section{Leitthemenitbersicht}

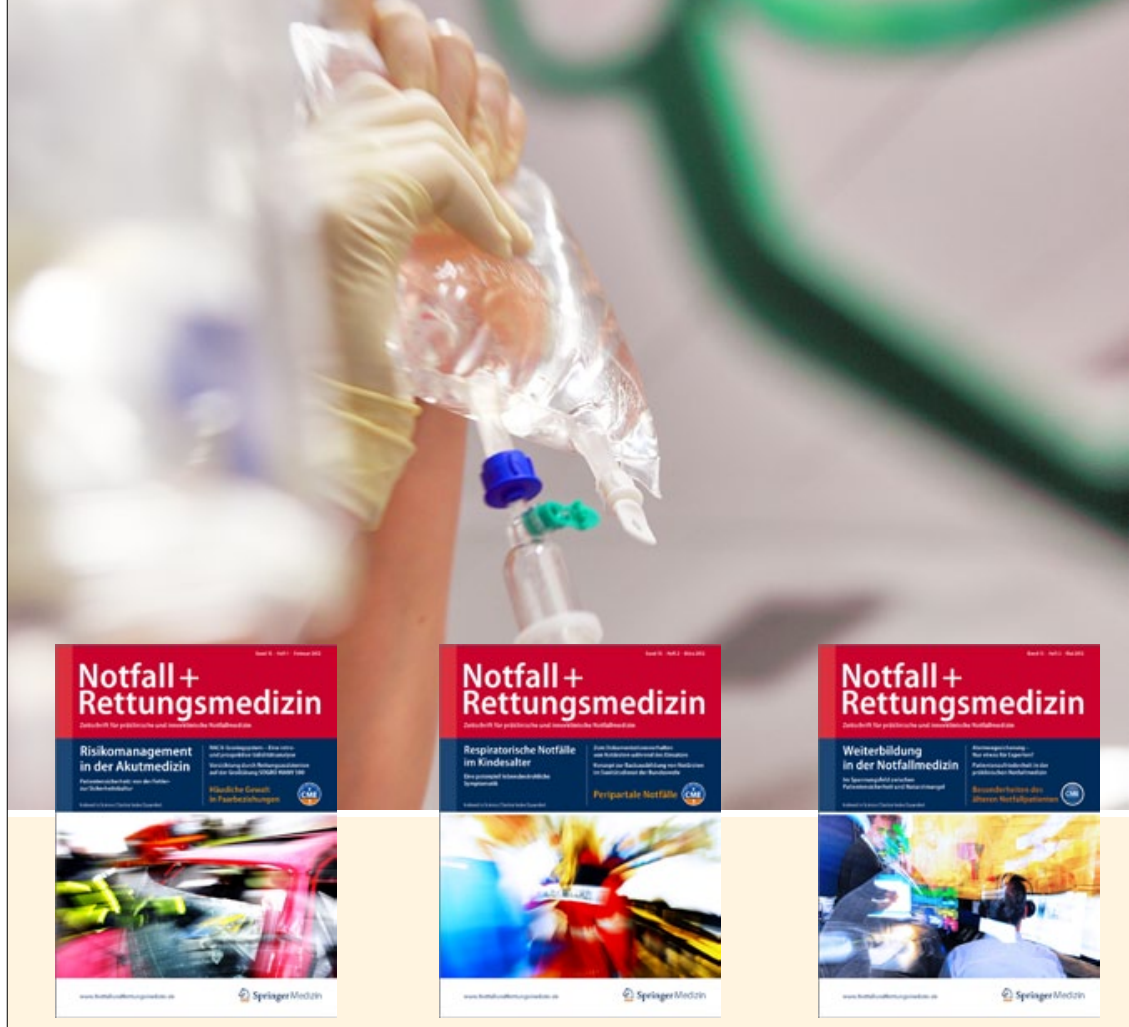

Die Notfall + Rettungsmedizin bietet Ihnen jeden Monat umfassende und aktuelle Beiträge zu interessanten Themenschwerpunkten aus allen Bereichen der Notfallmedizin. Möchten Sie ein bereits erschienenes Heft nachbestellen? Einzelne Ausgaben können Sie direkt bei unserem Kundenservice zum Preis von je EUR 35,- zzgl. Versandkosten beziehen:

\section{Überblick 2011}

1/11 Präklinisches Atemwegsmanagement

2/11 Lebensgefährliche Herzrhythmusstörungen

3/11 Notfallmedizin und Informationstechnologie

4/11 Leichte Schädel-Hirn-Traumata

5/11 Notaufnahme

6/11 Medizinrechtliche Aspekte der Notfallmedizin

7/11 Pädiatrische Notfälle

8/11 Advanced Technology in der Notfallmedizin

\section{Vorschau 2012}

1/12 Risikomanagement in der Akutmedizin

2/12 Respiratiorsche Notfälle im Kindesalter

3/12 Weiterbildung in der Notfallmedizin

4/12 Schnittstellen in der Notfallmedizin

5/12 Notaufnahme: Arbeitsplatz mit Zukunft

6/12 Kontroversen in der Reanimatologie

$7 / 12$ Intoxikationr

8/12 Ethik
So erreichen Sie unseren Kundenservice:

Springer Customer Service Center GmbH Kundenservice Zeitschriften

Haberstr. 7

69126 Heidelberg

Tel.: +49 $6221345-4303$

Fax: +49 6221 345-4229

E-Mail: leserservice@springer.com

\section{www.NotfallundRettungsmedizin.de}

